\title{
Inhibitory action of pristimerin on hypoxia-mediated metastasis involves stem cell characteristics and EMT in PC-3 prostate cancer cells
}

\author{
JIANWEI ZUO ${ }^{1 *}$, YUANQING GUO $^{2 *}$, XINSHENG PENG $^{1}$, YUBO TANG $^{3}$, XINTAO ZHANG $^{4}$, PEIHENG HE $^{1}$, \\ SHUAIHUA LI ${ }^{1}$, QINGDE WA ${ }^{1}$, JINGLEI LI ${ }^{5}$, SHUAI HUANG ${ }^{1}$ and DONGLIANG XU ${ }^{1}$

\begin{abstract}
${ }^{1}$ Department of Orthopaedic Surgery/Orthopaedic Research Institute, The First Affiliated Hospital of Sun Yat-Sen University; ${ }^{2}$ Department of Orthopaedic Surgery, The Fifth Affiliated Hospital of Sun Yat-Sen University; ${ }^{3}$ Department of Pharmacy, The First Affiliated Hospital of Sun Yat-Sen University, Guangzhou, Guangdong 510080; ${ }^{4}$ Department of Sports Medicine, Shenzhen Hospital of Peking University, Shenzhen, Guangdong 518036; ${ }^{5}$ Department of Radiology, Guangdong Academy
\end{abstract} \\ of Medical Sciences, Guangdong General Hospital, Guangzhou, Guangdong 510080, P.R. China
}

Received November 7, 2014; Accepted December 12, 2014

DOI: $10.3892 /$ or.2015.3708

\begin{abstract}
The aim of the present study was to investigate whether pristimerin affects the bone metastasis, stem cell characteristics and epithelial-mesenchymal transition (EMT) of prostate cancer ( $\mathrm{PCa}$ ) PC-3 cells subjected to hypoxia. The PC-3 cells were cultured under hypoxia or normoxia for $48 \mathrm{~h}$ and were then treated with increasing concentrations of pristimerin from 0 to $0.8 \mu \mathrm{mol} / 1$, under normoxia. Hypoxia-inducible factor- $1 \alpha$ (HIF- $1 \alpha$ ) was detected by western blotting. Proliferation was assessed with the CCK-8 assay. Transwell invasion assay was used to analyze the potency of invasion. Stem cell characteristics were detected by sphere formation, colony formation assay and western blotting, including CD44, KLF4, OCT4 and AGO2, which are stem cell characteristic-related markers. EMT was confirmed by the expression changes of EMT-related markers, including $\mathrm{N}$-cadherin, fibronectin, vimentin and ZEB1, which were evaluated by western blotting. The addition of pristimerin to the medium reduced the hypoxia-induced PC-3 cell proliferation in a dose-dependent manner. Pristimerin effectively inhibited
\end{abstract}

Correspondence to: Professor Dongliang Xu or Dr Shuai Huang, Department of Orthopaedic Surgery/Orthopaedic Research Institute, The First Affiliated Hospital of Sun Yat-Sen University, 58 Zhongshan 2nd Road, Guangzhou, Guangdong 510080, P.R. China

E-mail: dddrxu@163.com

E-mail: huang-shuai@hotmail.com

${ }^{*}$ Contributed equally

Abbreviations: PCa, prostate cancer; EMT, epithelial-mesenchymal transition; CSCs, cancer stem cells; Ago2, Argonaute 2; HIF-1 $\alpha$, hypoxia-inducible factor- $1 \alpha$

Key words: prostate cancer, bone metastasis, hypoxia, EMT, cancer stem cells hypoxia-induced invasion of the PCa cells in vitro. Moreover, the treatment of cells with pristimerin induced the reversal of hypoxia-induced stem cell characteristics and EMT, which was confirmed by sphere formation, colony formation assay and the expression changes of CSC- and EMT-related markers. The reversal of hypoxia-induced stem cell characteristics and EMT in the PCa cells by low-dose pristimerin was dose-dependent. These results showed that treatment with pristimerin may be a potential strategy for the suppression of hypoxia-induced metastasis through the reversal of hypoxia-induced stem cell characteristics and EMT in cancer cells, which justifies the potential use of pristimerin as a practical chemopreventive approach for patients with PCa.

\section{Introduction}

The formation of distant metastasis is the main cause of morbidity and mortality in patients with cancer(1). Once a solid tumor spreads to other tissues and organs, curative intervention with currently available cancer drugs, surgical operation and radiotherapy are ineffective. The principal characteristic of prostate cancer $(\mathrm{PCa})$ is its inclination to metastasize to the bones, which occurs in as many as $90 \%$ of the patients with advanced PCa (2). It appears that the bone microenvironment has a pivotal role in this process (3). Intratumoral hypoxia is a common pathophysiologic feature of solid tumors, from the smallest tumor of a few millimeters in diameter to the largest tumor (4). The hypoxic tumor environment results in aggressive and metastatic cancer phenotypes that are associated with resistance to radiation therapy, chemotherapy and a poor treatment outcome $(5,6)$. Hypoxia-inducible factor-1 (HIF-1) is the key factor in this process, regulating transcription of more than 70 hypoxia-activated genes, that range in function from those that promote anaerobic metabolism to those that initiate tumor metastasis (7). HIF-1 is a heterodimeric transcription factor composed of HIF-1 $\alpha$ and constitutively expressed HIF-1 $\beta$ subunits. Its biological activity depends on the amount of HIF-1 $\alpha$, which is tightly regulated by oxygen 
tension (8). Overexpression of HIF-1 $\alpha$ has been demonstrated in human cancers as compared with the respective normal tissues, making it a potential therapeutic target in oncologic drug discovery (9). Inhibition of HIF-1 $\alpha$ or its genetic disruption can not only block tumor cell growth, but also inhibit tumor metastasis, so that these tumor cells would be unable to respond to the hypoxic stimulus and progress $(10,11)$.

Emerging evidence indicates that CSCs may be the critical drivers of tumor progression and metastasis $(12,13)$. Somatic tumors, including PCa, contain a small subset of stem-like cells, called cancer stem cells, with capacities for self-renewal, differentiation and initiation of new tumors. Cancer stem cells preferentially reside in specific hypoxic microenvironment-niches, often existing inside tumors $(14,15)$. Meanwhile, stem-like cells are present in several established PCa cell lines, such as the PC-3 cell line (16) and under hypoxia possess greater stem-like properties (17).

Hypoxia, a negative prognostic factor for successful treatment, is a potent driver of a multitude of molecular signal pathways that allows cells to survive and thrive in the hostile tumor microenvironment and induces epithelial-mesenchymal transition (EMT) (18). EMT can be activated by hypoxia-induced HIF-1 signaling (19), followed by a cellular switch from epithelial to mesenchymal properties (20). EMT plays a key role in tumor cell metastasis (21) and has also been identified as an important step in bone metastasis of PCa (22,23). Hypoxia also provides tumor cells with cues for maintenance of a stem-like state and may help driving the linkage between EMT and CSCs (18).

Pristimerin is a quinonemethide triterpenoid with the potential of a promising anticancer agent and induces apoptosis in PCa cells (24), yet its activity in PCa under hypoxia has not been investigated. In light of these findings, we hypothesized that pristimerin affects the hypoxia-induced HIF-1 $\alpha$ expression and impair hypoxia-stimulated metastasis by inhibition of stem cell characteristics and EMT of PC-3 cells. To test this hypothesis, we first identified the anticancer efficacy of pristimerin in PC-3 cells under a hypoxic environment. Then, we evaluated the inhibitory effect of pristimerin on hypoxiainduced invasiveness of cancer cells. Furthermore, we investigated the reduction of the expression of HIF-1 $\alpha$ protein by pristimerin. We detected the stem cell characteristics of PC-3 cells by sphere formation and colony formation assay, and KLF4, OCT4 and AGO2 protein expression. In addition, EMT was evaluated by detecting $\mathrm{N}$-cadherin, fibronectin, vimentin, ZEB1 protein expression. Finally, our results supported that pristimerin exerted its repressive effect on the metastasis, at least in part, via restraining stem cell characteristics and EMT of PC-3 PCa cells. These findings provide further evidence that pristimerin can be a promising chemopreventive and anticancer agent in human cancer by inhibiting tumor metastasis under hypoxic stress.

\section{Materials and methods}

Reagents and antibodies. Pristimerin was purchased from Sigma-Aldrich Inc. (St. Louis, MO, USA). Pristimerin was solubilized in $100 \%$ dimethyl sulfoxide (DMSO) at $100 \mu \mathrm{g} / \mathrm{ml}$ concentration and frozen at $-20^{\circ} \mathrm{C}$ in small aliquots until needed. The antibody, anti-HIF-1 $\alpha$ was purchased from
BD Biosciences; CD44 and $\beta$-actin were obtained from Santa Cruz Biotechnology; and KLF4, OCT4, AGO2, N-cadherin, fibronectin, vimentin and ZEB1 were purchased from Cell Signaling Technology.

Cell lines and hypoxic treatment. Human PCa PC-3 cell lines were purchased from the American Type Culture Collection (ATCC; USA) and maintained in our laboratory for the present study. For conventional cell culture, cells were seeded in culture flasks with F-12 culture medium (HyClone) supplemented with $10 \%$ fetal bovine serum (FBS), $100 \mathrm{U} / \mathrm{ml}$ penicillin and $100 \mathrm{mg} / \mathrm{ml}$ streptomycin. All cells were cultured at $37^{\circ} \mathrm{C}$ in a humidified atmosphere and $5 \% \mathrm{CO}_{2}$ in air. For hypoxic exposure, the cells were placed in a sealed modular incubator chamber flushed with $1 \% \mathrm{O}_{2}, 5 \% \mathrm{CO}_{2}$ and $94 \% \mathrm{~N}_{2}$.

Cell proliferation assay. One day before the assay, cells were seeded under hypoxic or normoxic conditions for $48 \mathrm{~h}$, and were then displaced in 96-well plates in a volume of $100 \mu \mathrm{l}$ for $36 \mathrm{~h}$ and treated with increasing concentrations of pristimerin. All samples, including controls (normoxic), contained $0.1 \%$ DMSO. Viable cells were determined using CCK-8. The absorbance at $450 \mathrm{~nm}$ was determined using the ELx808 microplate reader (Bio-Tek, Winooski, VT, USA). Five wells were assayed at each concentration and the mean absorbance was determined. The data are expressed as mean \pm SD from three independent experiments.

Cell invasion assay. The migration and invasion assays were performed in a Transwell Boyden Chamber (Corning) using a polycarbonate filter with an $8-\mu \mathrm{m}$ pore size in a 24 -well plate and the filter membranes were coated with Matrigel (BD Biosciences) as previously described (25). One day before the assay, cells were seeded under hypoxic or normoxic conditions for $48 \mathrm{~h}$, and then cells were added to the inner chamber of the insert in $200 \mu \mathrm{l}$ of serum-free medium and $500 \mu \mathrm{l}$ of $10 \%$ FBS medium to determine the effect of pristimerin on cell invasion. Indicated concentrations of pristimerin were added to the lower chamber and all samples, including controls (normoxic), contained 0.1\% DMSO. After incubation under normoxia for $48 \mathrm{~h}$ at $37^{\circ} \mathrm{C}$, the cells were displated to the inner chamber of the insert in $200 \mu \mathrm{l}$ of serum-free medium for $24 \mathrm{~h}$, and then remained on the upper surface where they were gently removed using a cotton-tipped swab. The cells on the opposite surface of the filter membrane were stained with $0.1 \%$ crystal violet and counts were obtained from five randomly selected fields (x100 magnification) using a computer imaging system.

Spheroid formation assay. Spheroid formation assay was performed as previously described (26). To investigate the effect of pristimerin treatment in preventing hypoxia-induced spheroid formation, we maintained PC-3 cells under normoxic or hypoxic conditions for $48 \mathrm{~h}$, and then the cells were plated at $400 \mathrm{cells} /$ well onto 6-well poly-HEMA (Sigma)-coated plates and they were grown in F12 medium (HyClone) with increasing concentrations of pristimerin for 14 days supplemented with B27 (Invitrogen), $20 \mathrm{ng} / \mathrm{ml}$ EGF (Sigma) and $20 \mathrm{ng} / \mathrm{ml}$ basic FGF (Invitrogen). After 14 days, the number of prostaspheres (tight, spherical, non-adherent masses $>100 \mu \mathrm{m}$ in diameter) were counted, and images of the prostaspheres 
were captured under an inverse microscope. Sphere formation efficiency $=$ colonies/input cells $\times 100 \%$.

Colony formation assay. A colony formation assay was performed as previously described (26). To investigate the effect of pristimerin treatment in preventing hypoxia-induced colony formation, we treated PC-3 cells under normoxic or hypoxic conditions for $48 \mathrm{~h}$ and then treated them with indicative concentrations of pristimerin for $24 \mathrm{~h}$. After that the cells were plated at 300 cells as single cells onto a $65-\mathrm{mm}$ Petri dish with increasing concentrations of pristimerin for 14 days and colonies were stained with crystal violet. Plating efficiency $=$ number of colonies $(\geq 50$ cells/colony $) /$ input cells $\times 100 \%$. To determine different colony morphologies, the different colony morphologies were scored under a light microscope.

Western blot analysis. PC-3 cells were maintained under normoxic or hypoxic conditions for $48 \mathrm{~h}$, and were then treated by the indication concentrations of pristimerin for $24 \mathrm{~h}$ and cells were lysed with buffer containing $50 \mathrm{mM}$ Tris- $\mathrm{HCl}$ (pH 7.5), $5 \mathrm{mM}$ EDTA, $150 \mathrm{mM} \mathrm{NaCl}, 0.5 \%$ Triton X-100, $10 \mathrm{mM}$ sodium fluoride, $20 \mathrm{mM} \beta$-mercaptoethanol, $250 \mu \mathrm{M}$ sodium orthovanadate, $1 \mathrm{mM}$ phenylmethylsulfonyl fluoride and complete protease inhibitor cocktail (Sigma), and were incubated at $4^{\circ} \mathrm{C}$ for $30 \mathrm{~min}$. The lysates were ultrasonicated and centrifuged at $14,000 \mathrm{x}$ g for $15 \mathrm{~min}$. The proteins were separated on a $8-10 \%$ gradient SDS-PAGE and transferred onto nitrocellulose membranes (Hybond ECL; Amersham Pharmacia, Piscataway, NJ, USA). After being blocked with skimmed milk in 5\% TBST, the membranes were incubated overnight at $4^{\circ} \mathrm{C}$ with primary antibodies against HIF- $1 \alpha$, CD44, KLF4, OCT4, AGO2, N-cadherin, fibronectin, vimentin, ZEB1 or $\beta$-actin. After being washed for three times with TBST for $5 \mathrm{~min}$, the membranes were incubated with secondary anti-rabbit or anti-mouse antibodies for $1 \mathrm{~h}$ at room temperature and were then developed by ECL.

Statistical analysis. Data are shown as the means \pm SD. Multiple group comparison was performed by a one-way ANOVA for comparison of means. Comparisons between 2 groups were analyzed by the unpaired Student's t-test. P-values of $<0.05$ were considered to indicate a statistically significant result.

\section{Results}

Pristimerin inhibits the hypoxia-induced proliferation of $P C-3$ cells. Uncontrolled proliferation is one of the most distinguished traits of cancer cells. To determine the direct effect of pristimerin on hypoxic-induced cancer cell proliferation, we performed cell death assays on PC-3 cells treated with pristimerin (Fig. 1). Pristimerin treatment at a concentration of $0.4 \mu \mathrm{M}$ for $36 \mathrm{~h}$ led to inhibition of proliferation in the PC-3 cells and the effect was more marked at $0.8 \mu \mathrm{M}$. Our findings suggest that pristimerin inhibits the hypoxiainduced proliferation of cancer cells in a dose-dependent manner.

Pristimerin prevents hypoxia-induced invasion of $\mathrm{PC}-3$ cells. Hypoxia represents a physiological stimulus for tumor cell

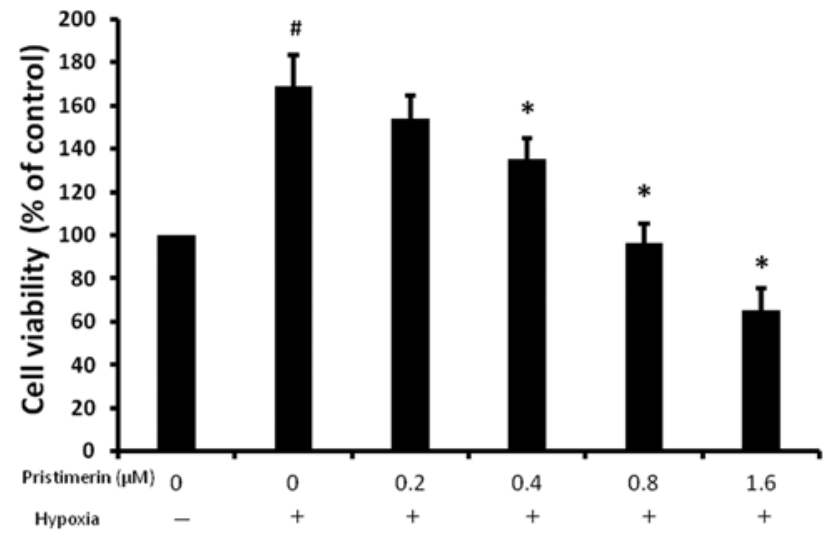

Figure 1. Pristimerin inhibits the hypoxia-induced proliferation of PC-3 cells PC-3 cells were exposed to hypoxic conditions for $48 \mathrm{~h}$, and then treated with the indicated concentrations of pristimerin. All concentrations included $0.1 \%$ DMSO as the control. After $36 \mathrm{~h}$, the cells were processed for the CCK-8 proliferation assay and the OD value was measured as described in Materials and methods. ${ }^{\#} \mathrm{P}<0.05$ vs. normoxia. ${ }^{*} \mathrm{P}<0.05$ vs. hypoxia only. DMSO, dimethyl sulfoxide; OD, optical density.

invasion and metastasis. The progression of tumors to an invasive phenotype and ultimately the formation of metastasis is associated with increased mortality (27). We examined whether pristimerin prevents the hypoxic-induced invasiveness of cancer cells by the Matrigel-coated Transwell assay. As shown in Fig. $2 \mathrm{~A}$ and B, an increase in the baseline invasiveness of the cancer cells was observed under hypoxic conditions as compared to normoxic conditions. The number of PC-3 cells that invaded through the Matrigel membrane increased from normoxia to hypoxia. The stimulatory effect of hypoxia on the invasiveness of cancer cells was reduced by pristimerin in a dose-dependent manner. The number of invaded cells under hypoxia decreased with the addition of $0.2 \mu \mathrm{M}$ pristimerin. These results indicate that pristimerin suppresses the hypoxiastimulated invasion ability of cancer cells.

Pristimerin prevents hypoxia-induced spheroid formation of $\mathrm{PC}-3$ cells. The ability to grow as non-adherent spheroids in sphere medium has been widely used to assess the self-renewal capability of CSCs and is one of the characteristics of prostate CSCs $(28,29)$. To investigate the effect of pristimerin treatment in preventing hypoxia-induced spheroid formation, we exposed PC-3 cells to normoxic or hypoxic conditions for $48 \mathrm{~h}$, and then the cells were pretreated with indicated concentrations of pristimerin for $24 \mathrm{~h}$. Next, the PC-3 cells were plated at 400 cells/well onto 6-well polyHEMA-coated plates and they were grown in F12 medium (HyClone). As shown in Fig. 3A and B, an increase in spheroid formation was observed under hypoxic conditions as compared to normoxic conditions. The stimulatory effect of hypoxia on the spheroid formation of cancer cells was reduced by pristimerin in a dose-dependent manner. The number of hypoxic invaded spheroids was decreased with the addition of $0.2 \mu \mathrm{M}$ pristimerin. These results indicate that pristimerin could suppress the hypoxia-stimulated spheroid formation ability of cancer cells.

Pristimerin prevents hypoxia-induced colony formation of $P C-3$ cells. To investigate the effect of pristimerin treatment in 

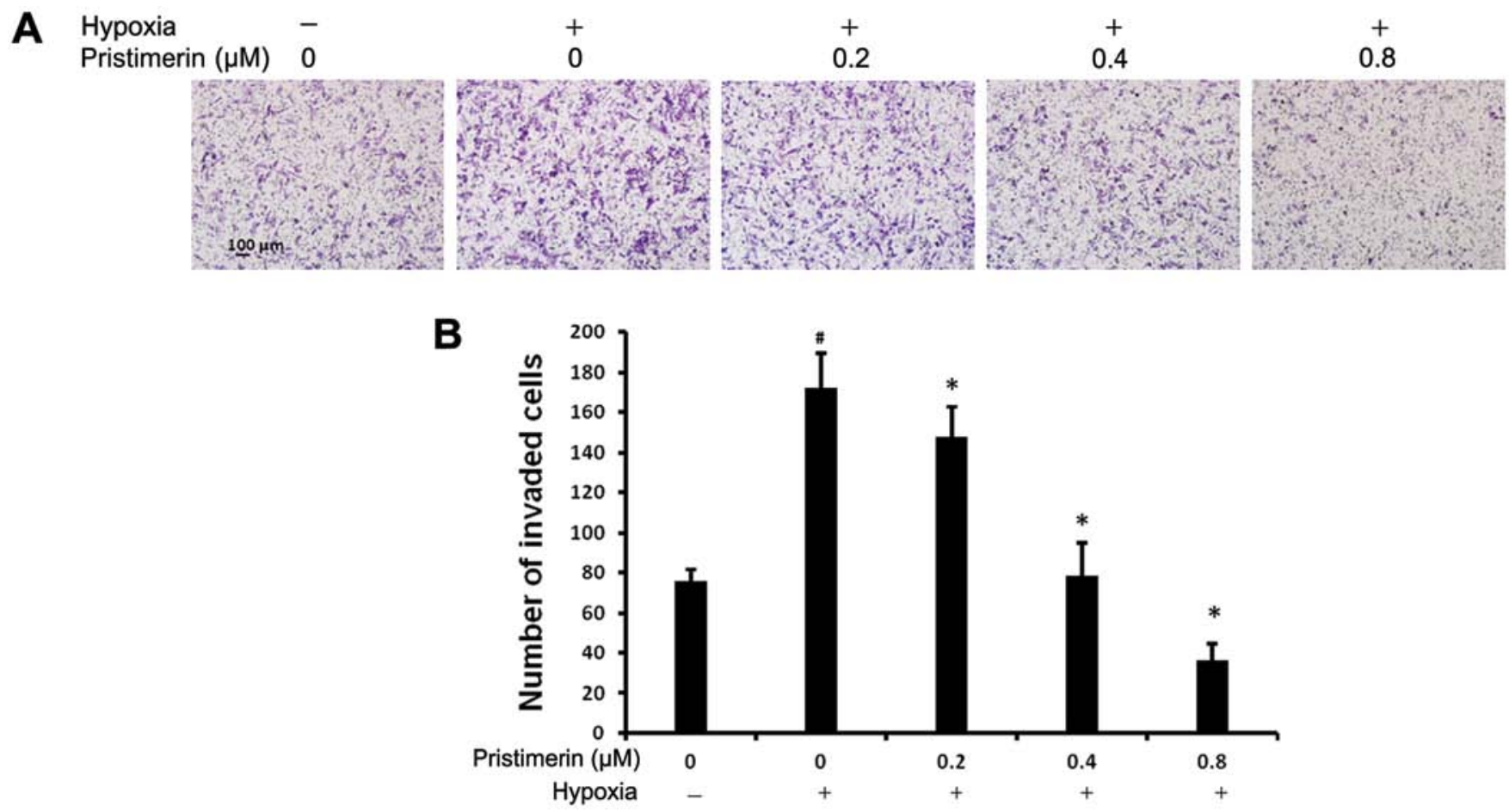

Figure 2. Pristimerin inhibits hypoxia-induced invasion of PC-3 cells. Pristimerin dose-dependently inhibited the invasion of PC-3 cells. (A) Images of invaded PC-3 cells (x100 magnification). (B) The PC-3 cells that had invaded to the lower chamber were counted in four randomly selected microscopic fields (x100 magnification). Values represent means $\pm \mathrm{SD}, \mathrm{n}=3 .{ }^{~} \mathrm{P}<0.05$ vs. normoxia. ${ }^{*} \mathrm{P}<0.05$ vs. hypoxia only.
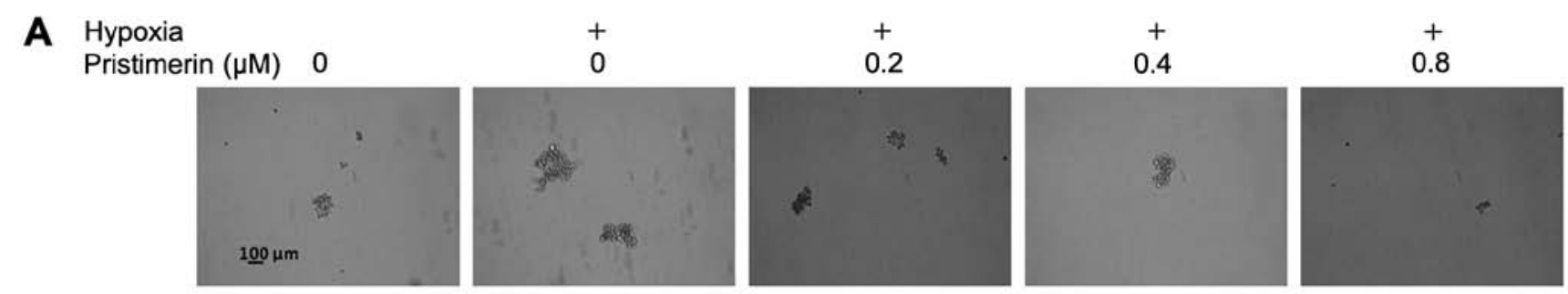

B

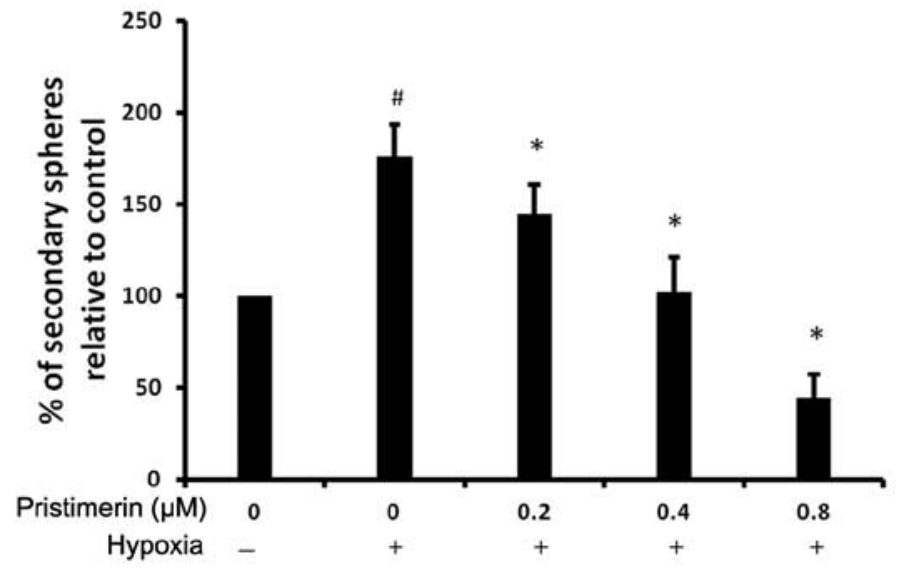

Figure 3. Pristimerin inhibits hypoxia-induced sphere formation of PC-3 cells. Pristimerin dose-dependently inhibited the sphere formation of PC-3 cells. (A) Images of sphere formation in PC-3 cells (x100 magnification). (B) The PC-3 cell sphere formation was counted in four randomly selected microscopic fields (x100 magnification). Values represent means $\pm \mathrm{SD}, \mathrm{n}=3 .{ }^{\text {" }} \mathrm{P}<0.05$ vs. normoxia. ${ }^{*} \mathrm{P}<0.05$ vs. hypoxia only.

preventing hypoxia-induced colony formation, we subjected the PC-3 cells to normoxic or hypoxic conditions for $48 \mathrm{~h}$, and then the cells were pretreated with the indicated concentrations of pristimerin for $24 \mathrm{~h}$. Next, they were plated at 300 cells as single cells onto a 6-well plate with increasing concentrations of pristimerin for 14 days. As shown in Fig. 4A and B, an increase in colony formation was observed under hypoxic conditions as compared to normoxic conditions. The stimula- 
A Hypoxia
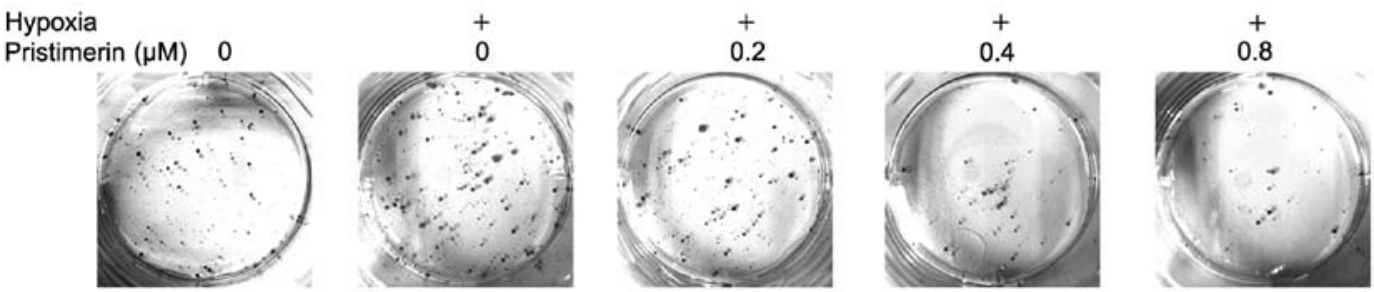

B

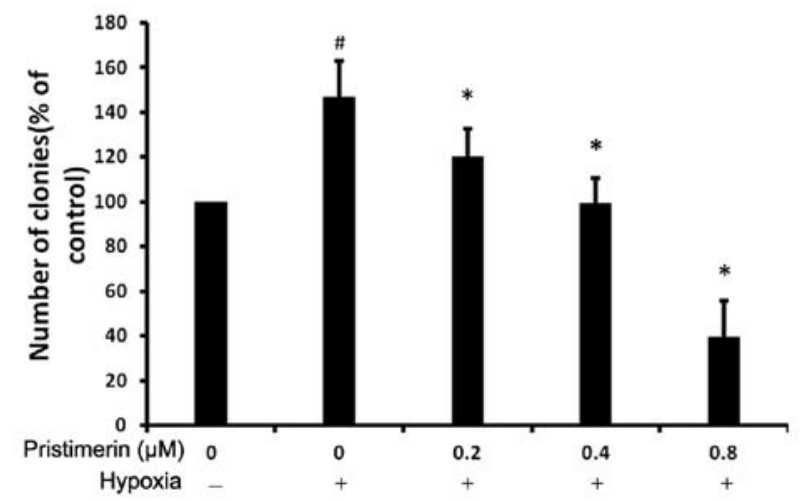

Figure 4. Pristimerin inhibits hypoxia-induced colony formation of PC-3 cells. Pristimerin dose-dependently inhibited the colony formation of PC-3 cells compared with control (normoxia). (A) Images of invaded PC-3 cells (x100 magnification). (B) The PC-3 cell colony formation was counted in four randomly selected microscopic fields (x100 magnification). Values represent means $\pm \mathrm{SD}, \mathrm{n}=3 .{ }^{*} \mathrm{P}<0.05$ vs. normoxia. ${ }^{*} \mathrm{P}<0.05$ vs. hypoxia only.

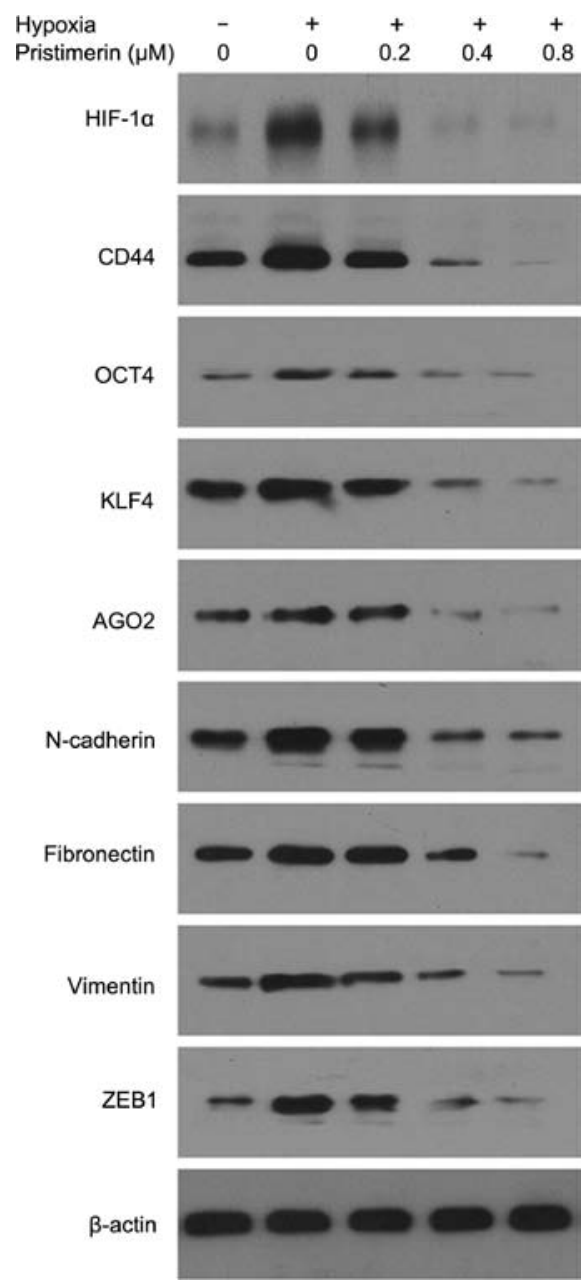

Figure 5. Pristimerin inhibits stem cell characteristics and EMT protein expression of PC-3 cells. PC-3 cells were exposed to normoxic or hypoxic conditions for $48 \mathrm{~h}$ and then treated with or without pristimerin for $24 \mathrm{~h}$. Pristimerin inhibited HIF-1 $\alpha$, CD44, OCT4, KLF4, AGO2, N-cadherin, fibronectin, vimentin and ZEB1 protein levels. tory effect of hypoxia on the colony formation of cancer cells was reduced by pristimerin in a dose-dependent manner. The number of hypoxic-induced colonies was decreased with the addition of $0.2 \mu \mathrm{M}$ pristimerin. These results indicate that pristimerin could suppress the hypoxia-stimulated colony formation ability of cancer cells.

Pristimerin prevents hypoxia-induced stem cell characteristics and EMT protein expression in PC-3 cells. To investigate the effect of pristimerin treatment in preventing hypoxiainduced stem cells, we subjected PC- 3 cells to normoxic or hypoxic conditions for $48 \mathrm{~h}$, and then the cells were pretreated with the indicated concentrations of pristimerin for $24 \mathrm{~h}$ and CD44, KLF4, OCT4, AGO2, N-cadherin, fibronectin, vimentin and ZEB1 levels were tested by western blotting. As shown in Fig. 5, an increase in CD44, KLF4, OCT4, AGO2, $\mathrm{N}$-cadherin, fibronectin, vimentin and ZEB1 expresssion was observed under hypoxic conditions as compared to normoxic conditions. The stimulatory effect of hypoxia on the expression of these proteins was reduced by pristimerin at a very low concentration $(0.2 \mu \mathrm{M})$. Hence, we showed that pristimerin treatment decreased CD44, KLF4, OCT4, AGO2, N-cadherin, fibronectin, vimentin and ZEB1 levels in a dose-dependent manner under hypoxia.

\section{Discussion}

In the present study, we found that pristimerin suppressed HIF-1 $\alpha$ expression and hypoxia-induced metastasis viablocking the proliferation, as well as the invasion ability of cancer cells. Furthermore, pristimerin repressed hypoxia-induced sphere formation, colony formation, expression of CSC markers and stemness factors including CD44, KLF4, OCT4, AGO2 and expression of EMT markers including $\mathrm{N}$-cadherin, fibronectin, vimentin, ZEB1 in PC-3 cells. 
These findings demonstrate that pristimerin can inhibit bone metastasis, hypoxia-induced stem cell characteristics and EMT of tumor cells. More importantly, CSCs and EMT may be the critical drivers of tumor progression and metastasis. Thus, our results suggest that the ability of pristimerin to inhibit bone metastasis is mediated partly by targeting hypoxia-induced stem cell characteristics and EMT of tumor cells.

Intratumoral hypoxia is a common characteristic feature of solid tumors and it is the driving force for tumor metastasis. Activation of hypoxia-inducible factor-1 (HIF-1) signaling is observed in a broad range of human cancers due to tumor hypoxia and promotes physiological changes associated with therapeutic resistance. It also includes the inhibition of apoptosis and senescence, as well as the activation of drug efflux and cellular metabolism, while approaches to inhibit HIF-1 signaling are primarily focused on reducing HIF- $1 \alpha$ protein levels (30). As a result, targeting HIF-1 represents an attractive strategy to enhance the efficacy of current therapies, as well as reduce resistance to chemotherapy in tumors (30). In the present study, pristimerin inhibited hypoxia-induced HIF-1 $\alpha$ protein levels. Therefore, our findings suggested that pristimerin may possess a similar function to hypoxia-induced progression and metastasis.

CSCs cells have been proposed to play a critical role in cancer metastasis as demonstrated in several human malignancies (31). With the affirmation of the CSC concept, cancer biology and cancer drug discovery have attained a new avenue to target cancer. CSCs are considered to be responsible for tumor initiation, metastasis and resistance to conventional radiotherapy and chemotherapy. Therefore, different approaches to targeting these tumorigenic and rare cells are urgently needed in order to improve the efficacy of anticancer therapy (32). In the present study, pristimerin inhibited hypoxia-induced stem cell characteristics and pristimerin directly inhibited the proliferation, sphere formation and colony formation of hypoxic PC-3 cells and impaired hypoxiainduced expression of CD44, KLF4, OCT4, AGO2 in hypoxic cancer cells. Therefore, our findings suggested that pristimerin may possess an inhibitory function against progression and metastasis by inhibited hypoxia-induced stem cell characteristics of PC-3 cells.

Increasing evidence emphasizes a critical role of EMT during PCa progression and malignant transformation, endowing the incipient cancer cells with invasive and metastatic properties (33-35). Thus, EMT could be a very promising therapeutic target, and the inhibition of EMT may prevent or restrain the invasion and metastasis of $\mathrm{PCa}$. We found that pristimerin could diminish the hypoxia-induced expression of $\mathrm{N}$-cadherin, fibronectin, vimentin, ZEB1 which showed that pristimerin changes the predominant mesenchymal phenotype. Therefore, our findings suggested that pristimerin may possess an inhibitory function against progression and metastasis by inhibition of EMT in PC-3 cells.

Due to the complexity of a tumor model, a single drug targeting a particular oncogene is unlikely to be entirely effective for cancer therapy. In this respect, pristimerin which contains various phytochemicals targeting multiple dysregulated pathways in cancer cells, may provide an alternative/complementary way to treat cancer. The utility of antitumor strategies for cancer control is strongly compromised by hypoxia-driven phenotypic changes, which make cancer cells more invasive and more prone to give rise to metastasis. In the present study, we showed that pristimerin decreased the expression of HIF-1 $\alpha$, CD44, KLF4, OCT4, AGO2, $\mathrm{N}$-cadherin, fibronectin, vimentin, ZEB1 in a dose-dependent manner under pre-treatment hypoxic conditions. Thus, it is evident that concurrent measures for halting hypoxia-induced phenotypic changes may be required if pristimerin therapy is ever to achieve its goal of providing long-term cancer control and improved survival.

In conclusion, we demonstrated that several activities of pristimerin may account for its suppressive effect on cancer metastasis under hypoxia. Firstly, pristimerin markedly inhibited the stimulatory effect of hypoxia on the proliferation and invasion ability of cancer cells. Secondly, pristimerin could suppress stem cell characteristics, which has been shown to regulate multiple steps in the complex process of metastasis. Thirdly, pristimerin decreased levels of the predominant mesenchymal phenotype, which is overexpressed in a variety of tumor types and associated with increased metastases and poor prognosis.

To the best of our knowledge, this is the first documentation that pristimerin exerts its broad spectrum of antimetastatic effects through its potent inhibition of stem cell characteristics and EMT, in the context of tumors under hypoxia, a common feature of most solid cancers. These results provide new insight into the mechanisms of the antitumor action of pristimerin.

\section{Acknowledgements}

This study was supported by grants from the National Natural Science Foundation of China (no. 81272938).

\section{References}

1. Duffy MJ, McGowan PM and Gallagher WM: Cancer invasion and metastasis: changing views. J Pathol 214: 283-293, 2008.

2. Carlin BI and Andriole GL: The natural history, skeletal complications, and management of bone metastases in patients with prostate carcinoma. Cancer 88 (Suppl 12): S2989-S2994, 2000.

3. Papachristou DJ, Basdra EK and Papavassiliou AG: Bone metastases: molecular mechanisms and novel therapeutic interventions. Med Res Rev 32: 611-636, 2012.

4. Höckel M and Vaupel P: Biological consequences of tumor hypoxia. Semin Oncol 28 (Suppl 8): S36-S41, 2001.

5. Greco O, Marples B, Joiner MC and Scott SD: How to overcome (and exploit) tumor hypoxia for targeted gene therapy. J Cell Physiol 197: 312-325, 2003.

6. Brizel DM, Scully SP, Harrelson JM, et al: Tumor oxygenation predicts for the likelihood of distant metastases in human soft tissue sarcoma. Cancer Res 56: 941-943, 1996.

7. Semenza GL: Targeting HIF-1 for cancer therapy. Nat Rev Cancer 3: 721-732, 2003.

8. Wang GL and Semenza GL: Purification and characterization of hypoxia-inducible factor 1. J Biol Chem 270: 1230-1237, 1995.

9. Zhong H, De Marzo AM, Laughner E, et al: Overexpression of hypoxia-inducible factor $1 \alpha$ in common human cancers and their metastases. Cancer Res 59: 5830-5835, 1999.

10. Blagosklonny MV: Hypoxia-inducible factor: Achilles' heel of antiangiogenic cancer therapy (Review). Int J Oncol 19: 257-262, 2001.

11. Maxwell PH, Dachs GU, Gleadle JM, et al: Hypoxia-inducible factor-1 modulates gene expression in solid tumors and influences both angiogenesis and tumor growth. Proc Natl Acad Sci USA 94: 8104-8109, 1997.

12. Chaffer CL and Weinberg RA: A perspective on cancer cell metastasis. Science 331: 1559-1564, 2011. 
13. Monteiro $\mathrm{J}$ and Fodde R: Cancer stemness and metastasis: therapeutic consequences and perspectives. Eur J Cancer 46: 1198-1203, 2010.

14. Marignol L, Coffey M, Lawler M and Hollywood D: Hypoxia in prostate cancer: a powerful shield against tumour destruction. Cancer Treat Rev 34: 313-327, 2008.

15. Sharifi N, Kawasaki BT, Hurt EM and Farrar WL: Stem cells in prostate cancer: resolving the castrate-resistant conundrum and implications for hormonal therapy. Cancer Biol Ther 5: 901-906, 2006.

16. Pfeiffer MJ and Schalken JA: Stem cell characteristics in prostate cancer cell lines. Eur Urol 57: 246-254, 2010.

17. Ma Y, Liang D, Liu J, et al: Prostate cancer cell lines under hypoxia exhibit greater stem-like properties. PLoS One 6: e29170, 2011.

18. Marie-Egyptienne DT, Lohse I and Hill RP: Cancer stem cells, the epithelial to mesenchymal transition (EMT) and radioresistance: potential role of hypoxia. Cancer Lett 341: 63-72, 2013.

19. Higgins DF, Kimura K, Bernhardt WM, et al: Hypoxia promotes fibrogenesis in vivo via HIF-1 stimulation of epithelial-to-mesenchymal transition. J Clin Invest 117: 3810-3820, 2007.

20. Thiery JP: Epithelial-mesenchymal transitions in development and pathologies. Curr Opin Cell Biol 15: 740-746, 2003.

21. Hanahan D and Weinberg RA: Hallmarks of cancer: the next generation. Cell 144: 646-674, 2011.

22. Nauseef JT and Henry MD: Epithelial-to-mesenchymal transition in prostate cancer: paradigm or puzzle? Nat Rev Urol 8 : 428-439, 2011.

23. Sethi S, Macoska J, Chen W and Sarkar FH: Molecular signature of epithelial-mesenchymal transition (EMT) in human prostate cancer bone metastasis. Am J Transl Res 3: 90-99, 2010.

24. Liu YB, Gao X, Deeb D, Arbab AS and Gautam SC: Pristimerin induces apoptosis in prostate cancer cells by down-regulating Bcl-2 through ROS-dependent ubiquitin-proteasomal degradation pathway. J Carcinog Mutagen (Suppl 6): 005, 2013.
25. Peng X, Guo W, Liu T, et al: Identification of miRs-143 and -145 that is associated with bone metastasis of prostate cancer and involved in the regulation of EMT. PLoS One 6: e20341, 2011.

26. Huang S, Guo W, Tang Y, Ren D, Zou X and Peng X: miR-143 and miR-145 inhibit stem cell characteristics of PC-3 prostate cancer cells. Oncol Rep 28: 1831-1837, 2012.

27. LiaoD, Corle C,Seagroves TN and Johnson RS: Hypoxia-inducible factor- $1 \alpha$ is a key regulator of metastasis in a transgenic model of cancer initiation and progression. Cancer Res 67: 563-572, 2007.

28. Visvader JE and Lindeman GJ: Cancer stem cells in solid tumours: accumulating evidence and unresolved questions. Nat Rev Cancer 8: 755-768, 2008.

29. Bisson I and Prowse DM: WNT signaling regulates self-renewal and differentiation of prostate cancer cells with stem cell characteristics. Cell Res 19: 683-697, 2009.

30. Warfel NA and El-Deiry WS: HIF-1 HIF-1 signaling in drug resistance to chemotherapy. Curr Med Chem 21: 3021-3028, 2014.

31. Hayashida T, Jinno H, Kitagawa Y and Kitajima M: Cooperation of cancer stem cell properties and epithelial-mesenchymal transition in the establishment of breast cancer metastasis. J Oncol 2011: 591427, 2011.

32. Duggal R, Minev B, Geissinger U, et al: Biotherapeutic approaches to target cancer stem cells. J Stem Cells 8: 135-149, 2013.

33. Xu J, Wang R, Xie ZH, et al: Prostate cancer metastasis: role of the host microenvironment in promoting epithelial to mesenchymal transition and increased bone and adrenal gland metastasis. Prostate 66: 1664-1673, 2006.

34. Whitbread AK, Veveris-Lowe TL, Lawrence MG, Nicol DL and Clements JA: The role of kallikrein-related peptidases in prostate cancer: potential involvement in an epithelial to mesenchymal transition. Biol Chem 387: 707-714, 2006

35. Kasper S and Cookson MS: Mechanisms leading to the development of hormone-resistant prostate cancer. Urol Clin North Am 33: 201-210, vii, 2006. 